\title{
Response to Letter to the Editor
}

We very much appreciate the thoughts and comments offered by Rady et al. (1). They note that (i) the measures of acculturation used in our analysis are interdependent and interrelated, which may influence measures of effect size and significance in our models and (ii) the measures may "oversimplify the complexity of the process of acculturation" (1).

With regard to the first point, our analyses did account, to the degree possible, for our surrogate measures being correlated and interrelated (length of US physical residence, US citizenship status, and English language proficiency). Factor analysis clearly demonstrated that two separable domains could be identified, with length of US physical residence and citizenship status forming one underlying factor/domain and English language proficiency (measured by three separate variables) forming a distinct second factor/domain. Using these two statistically separable surrogate measures of acculturation in our analyses was therefore an appropriate and statistically robust method for examining our research questions. Further, we successfully found that both measures of acculturation were significantly and independently associated with attitudes toward organ donation. Rady and colleagues are completely correct; however, when they note that it would be inappropriate to interpret the diminution of each variable's odds ratio as evidence of confounding (Table 3); it is merely evidence that each surrogate measure contributed some independent information to the overall model, reinforcing the strong overall finding that "greater acculturation into American society was associated with greater odds of believing organ donation to be justified" (2).

As for Rady and colleague's second point, we fully acknowledge that our measures of acculturation are surrogates and that they are highly useful measures but still limited markers of a complex psychological process. We would have of course preferred to have additional information, such as generational status, measures of exposure to a new culture, and other acculturation measures (3-5), but were limited to the variables included within the survey, which was constructed and conducted by others. However, although survey measures are by their nature limited to thin description and oversimplify complex psychological processes, such measures have consistently been demonstrated to be useful in studying acculturation. Detailed, contextually rich examinations of acculturation have been very valuable in understanding this psychology, but they are extremely resource intensive, and therefore, almost all the epidemiological literature of acculturation has come from studies such as ours. In fact, language proficiency is the most frequently studied domain of cultural changes within acculturation measures (5). Even given the admitted limitations of such surrogate measures, our study was still able to demonstrate that our acculturation measures were strongly and significantly associated with beliefs about organ donation.

The measures we utilized to represent the domain of psychological acculturation were guided by an established theoretical framework and obtained through rigorous exploratory factor analysis. There is considerable debate as to the optimal measures of acculturation, and to the underlying psychological domains, that should be measured through survey questions. Acculturation scales variably assess overt changes in daily life routines, subjective internal (attitudinal) changes, and at times simply measure exposure to a culture. For example, overt changes may be represented by language use or proficiency and food, entertainment, and media choices. Internal changes are often tested via questions pertaining to social values, cultural beliefs and senses of belonging to various social and ethnic groups. Generational status and demographic variables usually comprise the third type of scales. In sorting through the survey questions, we sought questions that fell within any of these aforementioned categories. We discovered five groupings of questions that related to acculturation: (i) Demographic Markers, (ii) English language proficiency, (iii) Measures of participation in ethnic activities and attachment to Arab community, (iv) Questions related to Arab identity, and (v) News media preferences. We next performed reliability checks using Cronbach's alpha and Kuder-Richardson coefficients and 
discarded individual items and domains with poor reliability. Our metric analyses (reliability check and factor analyses) suggested that combining all of these items did not lead to a reliable scale either. We then performed stepwise exploratory factor analyses using all remaining variables with sufficient reliability. Our analyses led to the discovery of 2 factors (eigenvalue $>1$ ), and we retained only those variables that had unique variances loading upon these two factors. These analyses led to the 2 measures of acculturation reported in our findings: (i) A measure of English language proficiency and (ii) a composite measure of length of residence in the United States and citizenship status. We then converted these measures into an index measure (Acculturation 1 index = a composite measure of length of residency in United States and US citizenship status) and an acculturation score (Acculturation 2 score $=$ English language proficiency).

Like Rady and colleagues, we strongly endorse the need for further research focusing on acculturation measures designed to better disentangle acculturation processes, attitudes, and ethical judgments regarding organ donation in the Arab American community.

\author{
Aasim I. Padela ${ }^{\mathrm{a}, \mathrm{b}}$, Shoaib Rasheed ${ }^{\mathrm{b}}$, \\ Gareth J.W. Warren ${ }^{\text {b, }}$, Hwajung Choi ${ }^{\text {b }}$ \\ and Amit K. Mathur ${ }^{\mathrm{d}}$ \\ ${ }^{\mathrm{a}}$ Department of Emergency Medicine, ${ }^{\mathrm{b}}$ Robert \\ Wood Johnson Foundation Clinical Scholars \\ Program, Department of Internal Medicine, \\ ${ }^{\mathrm{c}}$ Department of Urology, ${ }^{\mathrm{d}}$ Department of Surgery, \\ University of Michigan, Ann Arbor, MI, USA \\ e-mail: aasim@umich.edu
}

\section{References}

1. Rady M, Verheijde J, McGregor JL. Attitudes towards organ donation, moral pluralism, and acculturation in society. Clin Transplant 2011: 25: 809.

2. Padela Ai, Rasheed S, Warren GJW, Choi H, Mathur AK. Factors associated with positive attitudes toward organ donation in Arab Americans. Clin Transplant 2011: 25: 800.

3. Abdulrahim S, BAKER W. Differences in self-rated health by immigrant status and language preference among Arab Americans in the Detroit Metropolitan Area. Soc Sci Med 2009: 68: 2097.

4. Marin G, Sabogal $F$ et al. Development of a short acculturation scale for Hispanics. Hisp J Behav Sci 1987: 9: 183.

5. Matsudaira T. Measures of psychological acculturation: a review. Transcult Psychiatry 2006: 43: 462. 